\title{
MobiSeq: De Novo SNP discovery in model and non-model species through sequencing the flanking region of transposable elements
}

\section{Rey-Iglesia, Alba; Gopalakrishan, Shyam; Carøe, Christian; Alquezar-Planas, David E.; Nielsen, Anne Ahlmann; Röder, Timo; Bruhn Pedersen, Lene; Naesborg-Nielsen, Christina; Sinding, Mikkel-Holger S.; Fredensborg Rath, Martin}

Total number of authors:

16

Published in:

Molecular Ecology Resources

Link to article, DOI:

10.1111/1755-0998.12984

Publication date:

2019

Document Version

Peer reviewed version

Link back to DTU Orbit

Citation (APA):

Rey-Iglesia, A., Gopalakrishan, S., Carøe, C., Alquezar-Planas, D. E., Nielsen, A. A., Röder, T., Bruhn

Pedersen, L., Naesborg-Nielsen, C., Sinding, M-H. S., Fredensborg Rath, M., Li, Z., Petersen, B., Gilbert, M. T. P., Bunce, M., Mourier, T., \& Hansen, A. J. (2019). MobiSeq: De Novo SNP discovery in model and non-model species through sequencing the flanking region of transposable elements. Molecular Ecology Resources, 19(2), 512-525. https://doi.org/10.1111/1755-0998.12984

\section{General rights}

Copyright and moral rights for the publications made accessible in the public portal are retained by the authors and/or other copyright owners and it is a condition of accessing publications that users recognise and abide by the legal requirements associated with these rights.

- Users may download and print one copy of any publication from the public portal for the purpose of private study or research.

- You may not further distribute the material or use it for any profit-making activity or commercial gain

- You may freely distribute the URL identifying the publication in the public portal 
MS. ALBA REY DE LA IGLESIA (Orcid ID : 0000-0002-3768-9185)

MR. MIKKEL-HOLGER S. SINDING (Orcid ID : 0000-0003-1371-219X)

MR. ZHIPENG LI (Orcid ID : 0000-0001-8474-045X)

DR. MICHAEL BUNCE (Orcid ID : 0000-0002-7721-4790)

Article type : Resource Article

MobiSeq: De Novo SNP discovery in model and non-model species through sequencing the flanking region of transposable elements

Alba Rey-Iglesia ${ }^{1}$, Shyam Gopalakrishan ${ }^{1}$, Christian Carøe ${ }^{1}$, David E. Alquezar-Planas ${ }^{1,2}$, Anne Ahlmann Nielsen ${ }^{1}$, Timo Röder ${ }^{1}$, Lene Bruhn Pedersen ${ }^{1}$, Christina Næsborg-Nielsen ${ }^{1}$, Mikkel-Holger S. Sinding ${ }^{1,3}$, Martin Fredensborg Rath ${ }^{4}$, Zhipeng $\mathrm{Li}^{5}$, Bent Petersen ${ }^{6,7}, \mathrm{M}$. Thomas P. Gilbert ${ }^{1,8}$, Michael Bunce ${ }^{9}$, Tobias Mourier ${ }^{1,10}$, Anders Johannes Hansen ${ }^{1 *}$

${ }^{1}$ Natural History Museum of Denmark, University of Copenhagen, DK-1350 Copenhagen, Denmark.

${ }^{2}$ Australian Museum Research Institute, Australian Museum, Sydney, NSW, Australia

${ }^{3}$ Greenland Institute of Natural Resources, Kivioq 2, PO Box 570, 3900 Nuuk, Greenland

${ }^{4}$ Department of Neuroscience, University of Copenhagen, DK-2200 Copenhagen, Denmark ${ }^{5}$ Jilin Provincial Key Laboratory for Molecular Biology of Special Economic Animals, Institute of Special Animal and Plant Sciences, Chinese Academy of Agricultural Sciences, Changchun 130112, China

This article has been accepted for publication and undergone full peer review but has not been through the copyediting, typesetting, pagination and proofreading process, which may lead to differences between this version and the Version of Record. Please cite this article as doi: 10.1111/1755-0998.12984

This article is protected by copyright. All rights reserved. 
${ }^{6}$ DTU Bioinformatics, Department of Bio and Health Informatics, Technical University of Denmark, Lyngby, Denmark

${ }^{7}$ Centre of Excellence for Omics-Driven Computational Biodiscovery (COMBio), Faculty of Applied Sciences, AIMST University, Kedah, Malaysia.

${ }^{8}$ Norwegian University of Science and Technology, University Museum, Trondheim, Norway,

${ }^{9}$ Trace and Environmental DNA (TrEnD) Laboratory, School of Molecular and Life Sciences, Curtin University, Perth, Western Australia 6102, Australia

${ }^{10}$ Pathogen Genomics Laboratory, Biological and Environmental Sciences and Engineering Division, King Abdullah University of Science and Technology, Thuwal, Saudi Arabia

*Correspondence:

ajhansen@snm.ku.dk

Running title: MobiSeq: genotyping using TEs

Keywords: reduced representation library, transposable elements, TE, population genomics, genotyping, SNP discovery.

This article is protected by copyright. All rights reserved. 


\section{Abstract}

In recent years, the availability of reduced representation library (RRL) methods has catalysed an expansion of genome-scale studies to characterize both model and non-model organisms. Most of these methods rely on the use of restriction enzymes to obtain DNA sequences at a genome-wide level. These approaches have been widely used to sequence thousands of markers across individuals for many organisms at a reasonable cost, revolutionizing the field of population genomics. However, there are still some limitations associated with these methods, in particular, the high molecular weight DNA required as starting material, the reduced number of common loci among investigated samples, and the short length of the sequenced site-associated DNA. Here, we present MobiSeq, a RRL protocol exploiting simple laboratory techniques, that generates genomic data based on PCR targeted-enrichment of transposable elements and the sequencing of the associated flanking region. We validate its performance across 103 DNA extracts derived from three mammalian species: grey wolf (Canis lupus), red deer complex (Cervus sp.), and brown rat (Rattus norvegicus). MobiSeq enables the sequencing of hundreds of thousands loci across the genome, and performs SNP discovery with relatively low rates of clonality. Given the ease and flexibility of MobiSeq protocol, the method has the potential to be implemented for marker discovery and population genomics across a wide range of organisms - enabling the exploration of diverse evolutionary and conservation questions.

\section{Introduction}

Next generation sequencing (NGS) has revolutionized the world of genomics, allowing genome scale studies in model and non-model organisms (e.g. Davey et al., 2011; Ellegren, 2014; Goodwin, McPherson, \& McCombie, 2016). Despite ongoing cost reductions 
in both the sequencing of reference genomes, and the resequencing of genomes from individuals and populations (Fuentes-Pardo \& Ruzzante, 2017; Goodwin et al., 2016), it still remains cost-prohibitive for most research groups to generate whole genomes. Also, many research questions, such as population genomic or phylogenomic inferences, can be answered using a smaller set of SNPs that are measured in a subset of genomic regions (Davey et al., 2011). Thus, reduced representation library (RRL) methods have become a popular alternative for SNP discovery and genotyping (Davey \& Blaxter, 2011), in particular for nonmodel organisms. Several RRL strategies have been developed in the last years, including restriction site-associated DNA sequencing (RADseq) (Baird et al., 2008; Davey \& Blaxter, 2011), double digest RADseq (ddRADseq) (Peterson, Weber, Kay, Fisher, \& Hoekstram, 2012) or genotyping-by-sequencing (GBS) (Elshire et al., 2011), as well as the combination of RRLs with hybridization by capture for genotyping museum and ancient specimens (Sánchez Barreiro et al., 2016; Schmid et al., 2017).

The core feature of traditional RRL techniques is the use of restriction enzymes to obtain DNA sequence at a genome-wide set of loci (Andrews, Good, Miller, Luikart, \& Hohenlohe, 2016; Davey \& Blaxter, 2011). The sequencing of a subset of the genome by RRLs provides a high depth of coverage per locus at a reduced cost (Andrews et al., 2016), and to date RRLs have been successfully used in population genomic (e.g. Hohenlohe et al., 2010), phylogeographic (e.g. Emerson et al., 2010; Gaither et al., 2015), and phylogenomic studies (e.g. Wagner et al., 2013). Despite all the advantages that traditional RRL methodologies present, some important challenges remain: (1) high molecular weight DNA is required for enzymatic digestion, (2) allele dropout leads to high proportion of missing data, (3) high percentage of clonal reads, and (4) relatively complex laboratory workflows. Hybridization capture of RRL loci circumvents some of these challenges, allowing the sequencing of RRL loci in degraded samples and reducing allele dropout. However, bait 
design can be complex and costly, and typically requires commercially synthesized oligonucleotide probes (Faircloth et al., 2012; Sánchez Barreiro et al., 2016; Schmid et al., 2017). Thus, there is a niche for methods that allow some flexibility in the initial DNA quality requirements, while simplifying laboratory workflows, and reducing costs. In this study we present MobiSeq, a novel NGS genotyping method that takes advantage of the conserved sequences of transposable elements (TE) as anchoring points in order to generate sequence data containing the TE sequence, as well as the genomic regions flanking the element, which can be used for genotyping and SNP discovery. The method has been developed with the focus on samples stored or preserved in sub-optimal conditions that yield average DNA fragments between 350 - $800 \mathrm{bp}$.

TEs are self-replicating mobile elements that insert themselves in new places of the genome, either through a cut-and-paste or a copy-and-paste mechanism (Kazazian, 2004). The latter, also referred to as retrotransposable elements or type II elements, are predominant in mammalian genomes (with bats as a notable exception (Ray et al., 2008)). LINE elements are long retrotransposable elements encoding the enzymatic machinery required for their own movement. In contrast, SINEs are short transcribed sequences - often derived from small RNA genes - that do not encode any proteins, instead relying on proteins encoded by LINE elements (Dewannieux, Esnault, \& Heidmann, 2003). Insertions of LINEs and SINEs take place through the so-called target-primed reverse transcription, ensuring that the $3^{\prime}$ end of the elements are always present whereas the 5' may be truncated (Luan, Korman, Jakubczak, \& Eickbush, 1993). Most TEs display little insertion preference and can be scattered throughout the genome, although they are negatively selected in exonic regions (Sela, Mersch, HotzWagenblatt, \& Ast, 2010). They are found in almost all investigated eukaryotic genomes (Chénais, Caruso, Hiard, \& Casse, 2012) and typically constitute more than $50 \%$ of the genome in mammals (e.g. International Human Genome Sequencing Consortium, 2001; 
Sotero-Caio, Platt, Suh, \& Ray, 2016). The human and mouse genomes are by far the most well-studied animal genomes with regards to TE activity. Studies on these species have shown that the ongoing TE activity has resulted in genomic differences between closely related species (Mills et al., 2006; Yohn et al., 2005), populations (Akagi, Li, Stephens, Volfovsky, \& Simer, 2008; Cordaux, Srikanta, Lee, Stoneking, \& Batzer, 2007), and individuals (Konkel, Wang, Liang, \& Batzer, 2007; Levy et al., 2007).

Several studies have used NGS to investigate TEs, especially those associated with humans and diseases (e.g. Ewing \& Kazazian, 2010; Ewing \& Kazazian, 2011, Tang et al., 2016; Tubio et al., 2014,). The MobiSeq method presented here can generate many thousands of unique sequences per sample, each containing the TE sequence and the flanking genomic regions, which are subsequently used for SNP discovery and genotyping. MobiSeq relies on a modified version of the blunt-end single-tube double-stranded DNA library construction protocol described in Carøe et al. (2017), coupled with a TE-target enrichment PCR step prior to sequencing. TE-target primers can be designed to enrich for any TE element present in the species of interest, making it a very flexible protocol for use on eukaryotic genomic DNA (Figure 1). Furthermore, several TEs can be combined, in order to increase the number of sequenced markers, thus increase the proportion of genome coverage and analytical resolution.

To demonstrate efficacy, we report MobiSeq data on three mammal species: brown rat (Rattus norvegicus), grey wolf (Canis lupus lupus), and red deer complex species (Cervus $s p$ ). Two fallow deer samples (Dama dama) were also included to test the usefulness of the method when working with relatively distant species (e.g. red deer and fallow deer with a divergence date between 5 - 10 Myr (Lorenzini \& Garofalo, 2015)). Thus, we demonstrate its applicability and flexibility for genotyping model and non-model organisms, and discuss both possible future applications in the field of population genomics, and potential limitations.

This article is protected by copyright. All rights reserved. 


\section{Materials and methods}

\section{Sample information}

DNA was extracted from 55 brown rat, 20 grey wolf, and 28 deer samples, see Table S1 (Supporting Information) for a detailed description of the specimens included in the study. The deer samples used in the study were a mix of modern (1996 - 2015) and historic material (collected between 1909 - 1960). The rat sample set consisted of four male Sprague-Dawley rat individuals (Charles River Laboratories, Germany), each sampled from 13 different tissue types. Species selection was based on three criteria: 1) a model species with a wellcharacterized genome and TE variation in the genome (brown rat); 2) a species with good quality genome assembly available and studied extensively in population genetic studies (grey wolf) (e.g. Kardos et al., 2018; Pilot et al., 2014; Rutledge, Devillard, Boone, Hohenloge, \& White, 2015); and 3) a less well-studied complex of species (i.e. the red deer complex). DNA extractions were performed using DNeasy Blood and Tissue (Qiagen) following manufacturer's instructions. DNA elution was performed twice in $50 \mu 1 \mathrm{AE}$ buffer and with 10 minutes of incubation time at $37{ }^{\circ} \mathrm{C}$ prior to elution, in order to increase DNA yield. Extractions were quantified using a Qubit dsDNA High Sensitivity (HS) assay (Life technologies). Samples with higher DNA concentration than HS assay range were quantified again with a Qubit Broad Range assay (Life technologies).

\section{TE-target primer design}

In order to enrich our libraries for specific TE-target elements, we designed four TE primers for repeat families that showed hallmarks of recent activity. From the RepeatMasker (Smit, Hubley, \& Green, 2013) annotations of the rat and the dog genome (Speir et al., 2015; Rat Genome Sequencing Consortium, 2014; Lindblad-Toh et al., 2005), we extracted 3' tails from TE loci that were nearly full-length and showed low levels of divergence from the consensus sequence. Sequences were extracted using samtools (Li et al., 2009) and aligned 
with muscle (Edgar, 2004). Highly conserved regions were determined from the alignments, and selected as potential primer sites. This resulted in one primer targeting a L1 TE (a superfamily of LINEs) in brown rats (L1RnT1: 5' CCGGAAACCGGGAAAGGGAATAACAC 3'); and two primers targeting two different TE in grey wolves (SINE: 5'GAGACCCGGGATCGAATCCC 3'; LINE: 5' GATAGCCAAACTGTGGAAGG 3'). Due to the lack of a well-annotated genome for the red deer complex, a SINE primer was selected from the study Nilsson, Klassert, Bertelsen, Hallström and Janke (2012); (BOV2A: 5' GGGACGGGGGAGCCTGGTGGGCTG 3'). The TE-target oligonucleotides were then combined with the sequence of the P7 adapter (Meyer \& Kircher, 2010), in order to create a fusion primer (TE+P7) that would enrich for TE sites, at the same time as adding the P7 sequencing adapter adapter compatible with binding to Illumina flow cells (see Table S2 for an overview of the oligonucleotides).

\section{Modified P5 adapter}

A modified version of the P5 adapter from Meyer and Kircher (2010) was designed for this protocol. In this modified P5 (mp5), the IS1 oligo is kept as in Meyer and Kircher (2010). However, IS3 oligonucleotide presents a modified sequence by adding a C3 spacer at the 3' end blocking polymerase extension. This, together with the conventional lack of a 5'phosphate, allows us to run a PCR reaction using a universal primer for the adapter sequence (IS4 or IS7, see Table S2, Supporting Information) and a TE-target primer enriching for a specific subset of TEs (Figure 1). Hybridization of IS1 and the modified IS3 to generate mp5 was performed as in Meyer and Kircher (2010).

This article is protected by copyright. All rights reserved. 


\section{Methods for preparation of sequencing libraries}

An overview of the method is represented in Figure 1 (full protocol available as Appendix S1, Supporting information). Prior to library build, DNA extracts were fragmented using Bioruptor NGS (Diagenode) to an average length of 600 - $700 \mathrm{bp}$. Four of the samples were not fragmented prior to library build, as their average DNA fragment size was ca. 350 $400 \mathrm{bp}$ (Table S1, Supporting information). Starting material for Bioruptor varied across samples (between 200 - $2000 \mathrm{ng}$ ), depending on the DNA extract concentration (Table S1, Supporting information). Biorupted DNA was size selected using magnetic beads purification, either Agencourt AMPure XP (Beckman Coulter) or Sera-Mag Speedbeads (ThermoScientific) at $0.7 x$ to remove DNA fragments shorter than ca. $200 \mathrm{bp}$. Size selected DNA was then quantified using Qubit HS reagents, as previously described, and loaded in a TapeStation High Sensitivity (TS-HS) 2200 (Agilent) to obtain a length profile of the fragmented material. Illumina library building was based on a recently developed blunt-end single-tube protocol (Carøe et al., 2017) with some modifications as in Mak et al. (2017) (full protocol available as Appendix S1, Supporting information). In particular, the ligated adapters differed from the ones in the original protocol by excluding the use of a P7 adapter (only using the mp5 adapter) and excluding the adapter fill-in reaction. Following library preparation, the reactions were purified using a magnetic beads purification protocol, Agencourt AMPure XP (Beckman Coulter) or Sera-Mag Speedbeads (ThermoScientific), at 0.70x. Purified libraries were eluted in $30 \mu 1$ of EBT.

\section{TE-enrichment PCR}

Libraries were enriched for fragments containing the TE of interest by using a TEenrichment PCR. Primers for this PCR were forward primer IS4 or IS7 (Meyer \& Kircher, 2010) and the fusion reverse primer described in prior sections (Table S2 for oligonucleotide 
sequences, Supporting information), see Figure 1 (TE-enrichment PCR). PCR reactions were performed in $25 \mu$ containing: 1x Accuprime Pfx Mix (ThermoScientific), $0.4 \mu \mathrm{M}$ of each primer, 2\% DMSO (ThermoScientific), $0.02 \mathrm{U} / \mu \mathrm{l}$ Accuprime Pfx polymerase, and $5 \mu \mathrm{l}$ of the purified libraries. Cycling parameters were denaturation at $95^{\circ} \mathrm{C}$ for 30 seconds, followed by 10-15 cycles of denaturation at $95{ }^{\circ} \mathrm{C}$ for 30 seconds, annealing between $60-67{ }^{\circ} \mathrm{C}$ (depending on the TE-target primer) 30 seconds, and extension at $68{ }^{\circ} \mathrm{C}$ for 60 seconds). TEenriched libraries were purified using magnetic beads at 1x. Purified DNA was eluted in 30 $\mu \mathrm{l}$ of EBT. Concentration was measured using a Qubit HS assay and loaded in a TS-HS 2200 (Agilent).

\section{Index PCR and sequencing}

TE-enriched libraries were indexed and amplified for sequencing as described in Meyer and Kircher (2010). PCR reactions were performed in $25 \mu$ l containing: 1x Accuprime Pfx Mix (Thermo Fisher), $0.4 \mu \mathrm{M}$ of each primer, 2\% DMSO, $0.02 \mathrm{U} / \mu \mathrm{l}$ of the enzyme, $5 \mu \mathrm{l}$ of the purified libraries. Cycling parameters were denaturation at $95{ }^{\circ} \mathrm{C}$ for 30 seconds, followed by $5-10$ cycles of (denaturation at $95{ }^{\circ} \mathrm{C}$ for 30 seconds, annealing at $60{ }^{\circ} \mathrm{C}$ for 30 seconds, and extension at $68{ }^{\circ} \mathrm{C}$ for 60 seconds). Indexed libraries were purified using magnetic beads at $1 \mathrm{x}$. Purified DNA was eluted in $30 \mu \mathrm{l}$ of EBT. Concentration was measured using a Qubit HS assay and loaded in a TS-HS 2200 (Agilent). Indexed libraries were pooled and sequenced at the Danish National High-throughput Sequencing Centre, Copenhagen, Denmark, on an Illumina MiSeq Instrument for 250 cycles in paired-end read mode. The sequencing architecture is illustrated in Figure 2.

This article is protected by copyright. All rights reserved. 


\section{Data processing}

Raw reads were filtered based on the presence of the TE-target primer sequence in the 5'end of the reverse R2 (Figure 2, Supplementary Figure 1). Reads without the TE-target primer sequence were discarded, as they represent off-target amplifications or PCR artefacts. Reads derived from the rat samples were merged by individual (see Table S1, Supporting information) and treated as such in all downstream analyses. Paired-end filtered reads were trimmed of adapter sequences, reads shorter than 25 bp were discarded and quality filtered using PALEOMIX (Schubert et al., 2014). Retained paired-end reads were mapped against a reference genome of the different species included in the study: brown rat genome (GCA_000001895.4; Rat Genome Sequencing Consortium, 2014); wolf genome (Gopalakrishnan et al., 2017); and red deer genome (Zhipeng Li et al., unpublished). Mapping was performed with BWA (Li \& Durbin, 2010) using the mem algorithm and softclipping. PCR duplicates and reads mapping to multiple genomic locations were marked using Mark Duplicates (https://broadinstitute.github.io/picard/). GATK (DePristo et al., 2011) was used to perform indel realignment. Basic sequencing statistics, such as read numbers and clonality were quantified within the PALEOMIX pipeline (Schubert et al., 2014). For all downstream analysis, only reads mapping to loci (TE sites) that occur in more than $90 \%$ of the samples were retained using the bedtools software suite (Quinlan \& Hall, 2010). The pairwise coverage comparisons for the retained set of loci were estimated for each primer pair using samtools bedcov (Li et al., 2009).

Aggregate coverage plots were generated for each sample and dataset using agPlus (Maehara \& Ohkawa, 2015) from the primer site up to $1 \mathrm{~kb}$ of the flanking region. The total coverage was used to correct for sequencing depth differences between samples. Forward and reverse reads derived from (+) and (-) strand sequencing were merged into one plot, as well as analysed separately to detect strand-associated biases (Figure 2). Average GC content (\%) 
per loci was estimated across datasets to explore the variation of coverage in relation to GC content across loci. PRESEQ (Daley \& Smith, 2013) was used to infer the complexity of each library for all the sequenced loci.

ANGSD (Korneliussen, Albrechtsen, \& Nielsen, 2014) was used to perform SNP calling, requiring a minimum mapping quality of 30 , minimum base quality of 20 , minimum depth of coverage of $3 x$ per genotype, and coverage in at least $50 \%$ of individuals. NgsDist (Vieira, Lassalle, Korneliussen, \& Fumagalli, 2015) was used to estimate the pairwise distances between the samples for each dataset using the SNP data generated with ANGSD. Trees were estimated from pairwise genetic distances with 100 bootstrap replicates using FastME (Lefort et al., 2015) and RaxML (Stamatakis, 2014, https://github.com/amkozlov/raxml-ng).

\section{Results}

Number of sequenced loci, coverage, depth and clonality

Our sequencing yielded a total of $45,255,518$ raw reads. An average of $95 \%$ of the reads across datasets contained the TE-target primer in R2. Less than $1 \%$ of the reads were discarded after trimming adapters and quality filtering, and more than $99 \%$ of the remaining reads were mapped to each specific genome. Between $24 \%$ and $0.4 \%$ of the reads were discarded after duplicate removal. One of the deer specimens presented a higher number of PCR duplicates than average (see Discussion). See Table 1 and Table S3 (Supporting information) for more detailed sequencing and mapping statistics.

Our sequencing strategy yielded a variable number of TE-enriched loci across genomes, depending on the TE-target primer used for the different species. Note that for number of loci estimations and downstream data analyses, BOV2A was divided in two datasets (1) BOV2A_all including all the cervid samples, and (2) BOV2A_CE including only 
Cervus sp. (Table S1, Supporting information). Table 2 summarizes all the TE-loci identified, as well as those found in at least $90 \%$ of the samples. In all subsequent downstream analyses, only the loci at the $90 \%$ cut-off were used.

We performed pairwise comparisons of the coverage at primer sites across the $90 \%$ cut-off loci across the different datasets. Supplementary Figures 2 - 5 represent the $90 \%$ cutoff loci pairwise comparisons. Wolf_LINE and Wolf_SINE, and Rat_L1 showed high primer coverage correlation across samples, which suggests that sequencing across loci and samples was similar. BOV2A presented more variation in primer coverage, with a few samples showing very low pairwise correlations to the rest of the samples.

Aggregate coverage plots show that the highest coverage values are derived from the $250 \mathrm{bp}$ to $0 \mathrm{bp}$ of the distance from the primer site (Figure 3). This region represents all the $\mathrm{R} 2$ reads carrying the TE-target primer sequence. The slight increase in coverage before 0 is derived from R1 reads that overlap with R2. This is correlated with DNA fragment length and the sequencing mode used in this study, MiSeq 250 PE (i.e. short DNA fragments will result in high amount of overlap between the two reads in the read pair). The shorter average size in the BOV2A material clearly reflects this (Figure 3). After position 0, that represents the primer site, there is a drop in coverage that will continue decreasing, as the sequencing moves into the flanking region (Figure 3). Depth of coverage was variable between datasets; in general, the highest coverage values were derived from the wolf LINE dataset. The recovered flanking region was variable between datasets and it is influenced by initial DNA fragment size. BOV2A aggregate plot serves as an example of this. Supplementary Figures 6 - 9 show aggregate coverage plots split by (+) and (-) strand.

This article is protected by copyright. All rights reserved. 
SNP discovery and genotyping

The sequenced TE-enriched loci yielded variable number of SNPs for the different TE-target primers. The total number of SNPs detected using ANGSD was: Wolf_LINE = 68,961; Wolf_SINE $=81,292 ;$ BOV2A_all $=210,949 ;$ BOV2A_CE $=114,973$; and Rat_L1 = 64,482. We explored the variability in SNP numbers in relation to the minimum number of individuals sharing SNPs (Figure 4). The general trend is an increase in the number of shared SNPs as the number of individuals increases. However, we detected a drop in the number of shared SNPs for some of the primer pairs: Wolf_SINE (from 9 to 10 individuals), BOV2A_all (from 26 to 28 individuals), and BOV2A_CE (from 24 to 26 individuals). This drop seems to be associated with samples that performed poorly in the TE-enrichment PCR. We also investigated minor allele frequencies in the wolf and deer datasets (the rat dataset was excluded from these analyses because of the low number of samples) (Figure 4). The dataset BOV2A_CE presented a larger number of rare alleles than the other datasets.

We investigated cumulative rate of loci and SNPs sequenced per Megabase across genome scaffolds for each dataset (Supplementary Figure 10). Our results indicate that MobiSeq TE-target PCR amplified loci across all the scaffolds in the genome assembly, thus allowing us to perform random genone-wide SNP discovery across datasets. Finally, we explored SNP distribution with respect to the distance from the primer site (Supplementary Figure 11). Our results show that as sequencing moves into the flanking region, the number of discovered SNPs decreases. We note that the pattern of the number of SNPs discovered is reflective of the pattern of coverage across the loci (Figure 3). As an example of the applicability of MobiSeq data to estimate evolutionary relationships, we generated NGS distance trees based on SNPs for the wolf and deer datasets (Supplementary Figures 12 - 14).

This article is protected by copyright. All rights reserved. 


\section{GC content and coverage}

We explored the relationship between depth of coverage and GC content of the sequenced loci (Figure 5). Average GC content (\%) in the sequenced loci is similar to the average GC content in the respective mammalian genomes. Except for the BOV2A_all dataset, the highest coverage is usually derived from the regions with around $45 \% \mathrm{GC}$ content and it drops for windows with GC content more than 50\%-55\%.

\section{Library complexity}

We used PRESEQ to estimate and predict the complexity of the MobiSeq libraries across all TE-target primers and samples (Figure 6). Our results indicate that MobiSeq libraries were sufficiently complex and that a sequencing plateau was not reached.

\section{Discussion and conclusions}

In this study, we have demonstrated the feasibility and adaptability of MobiSeq in three different taxa across a variety of tissues using both modern and degraded historical DNA.

\section{Amount of input DNA needed and Quality}

Compared to other RRL protocols, the DNA quality and input requirements for MobiSeq offers a degree of flexibility. In general, traditional RRL methods require high molecular weight genomic DNA, which represents a limitation for poorly preserved samples (Andrews et al., 2016). In MobiSeq, highly fragmented material would result in fragmentation of the TE-target primer sites, which would reduce PCR success and affect the number of targeted loci. Also, the DNA quality of the starting material will have an influence on the length of the flanking region (i.e. highly fragmented DNA will reduce the sequenced flanking region). The historic deer specimens included in this study yielded an average DNA fragment size around $350 \mathrm{bp}$ in the starting material (Table S1, Supporting information). 
Despite the underperformance of these samples in terms of clonality, coverage, and length of the flanking region, library complexity, and amount of missing data, we were still able to recover sufficient informative genomic data to include them in our NGS distance-based trees (Supplementary Figure 12). Nevertheless, whenever possible, we would suggest average DNA fragment sizes between $700-800 \mathrm{bp}$ as, empirically, this is the material that has yielded the optimal results (i.e. number of sequenced SNPs and loci) in our analyses.

DNA input amount can be highly variable. We would suggest building the library on at least $100 \mathrm{ng}$ of total input DNA. The current required DNA input in MobiSeq is similar to the amount used in RAD methods (Andrews et al., 2016). However, ongoing experiments indicate that starting material can be reduced down to $50 \mathrm{ng}$ (Rey-Iglesia et al., unpublished).

\section{Duplicates}

One of the limitations of RRL methodologies is the clonality generated due to PCR artefacts that would lead to increased artificial coverage and would affect SNP calling. For instance, several studies reported that PCR duplicates occur at high frequencies in RADseq data (e.g. Andrews \& Luikart, 2014; Schweyen, Rozenberg, \& Leese, 2014). PCR duplicates can be identified in RAD protocols that include a random shearing step and paired-end sequencing, like the original RADseq (Andrews et al., 2016; Davey et al., 2013). However, PCR duplicates cannot be identified in some of the other RAD strategies, because all fragments for a given locus will have identical start and stop positions (Andrews et al., 2016; Davey et al., 2011). Alternatives for controlling PCR duplicates are using PCR-free protocols, such as ezRAD that relies on Illumina PCR-free kits for library build. However, PCR-free methods are typically more expensive and require large amounts of starting material (Andrews et al., 2016).

This article is protected by copyright. All rights reserved. 
In MobiSeq, the random shearing of the DNA prior to library build and the TE-target PCR set up generates fragments with different starting points in the 5 ' start of the flanking region. This allows identification of putative PCR duplicates, based on the assumption that any read pair with identical starting position of the paired-end read are duplicates. However, in general, we obtained low values of clonality across datasets (Table 1, Table S3, Supporting information). Only one of the samples included in the study, a specimen from the BOV2A dataset, presented clonality values $>20 \%$. The low clonality values obtained are associated with the high complexity of the libraries, due to the abundance of the targeted elements in the genome and the amplification success of our TE-target PCR approach.

\section{Depth of coverage}

The highest depth of coverage was detected in the region closest to the primer site (ca. $250 \mathrm{bp}$ representing the length of $\mathrm{R} 2$ ), coverage decreases as sequencing moves into the flanking region. Nevertheless, the average depth of coverage across the loci allowed for robust SNP calling using standard variant calling methods, as well as likelihood methods, such as those implemented in ANGSD.

Three principal factors that could be influencing the average depth of coverage are (i) high numbers of the targeted TEs, (ii) the sequencing strategy, and (iii) GC content of the sequenced loci. We sequenced our libraries using Illumina MiSeq 250 PE chemistry, obtaining an average number of reads per sample ranging from 431,916 in wolf_LINE to 1,695,644 in BOV2A. Higher numbers were recovered for rats (Table 1, Table S2, Supporting information), as each individual represents a pool of 13 libraries. A study on Canis admixture using RADSeq reported raw reads numbers between 8 - 37 million per sample (Rutledge et al., 2015). Other studies report average values of raw read numbers between (2 - 3 million reads) using RADSeq (e.g. Lah et al., 2015; Skovrind et al., 2016). 
Considering the total number of TE loci per dataset and the PRESEQ results, we would suggest using sequencing platforms with higher throughput (e.g. Illumina HiSeq 4000) to increase the amount of generated data, as well as depth of coverage. This would also allow us to obtain a more accurate comparison of MobiSeq with other RRL methodologies. The depth of coverage seems to also be influenced by the GC content of the sequenced loci. In general, our results show that regions containing around $45 \%$ GC content present the highest depth of coverage.

\section{Allele dropout}

RRL datasets are likely to contain high proportions of missing data, mostly due to polymorphisms in the restriction enzyme recognition site (Gautier et al., 2013). In RRL methods, when a polymorphism occurs at a restriction enzyme recognition site, the enzyme will fail to cut the genomic DNA at that location, leading to allelic dropout (Andrews \& Luikart, 2014; Andrews et al., 2016; Gautier et al., 2013;). Thus, even though high numbers of loci are sequenced per sample, the number of comparable sites can become highly reduced (Gautier et al., 2013). We believe that allelic dropout in MobiSeq is mostly dependent on DNA degradation, PCR biases, and distribution of the TE elements. The percentage of retained loci varied widely in the different datasets. The amount of loci shared by at least $90 \%$ of the individuals in both wolf datasets was around $25 \%$, while in the cervid datasets it was around $4 \%$. The low percentage of shared loci in the cervid datasets might be associated with the variable levels of DNA degradation and the distribution of the targeted TE in cervid genomes. BOV2A, the TE targeted in the cervid dataset, is a SINE element that is widely

distributed in the genomes of ruminants (e.g. deer) (Nilsson et al., 2012; Onami, Nikaido, Mannen, \& Okada, 2007). The high abundance of the element combined with the reduced effort on sequencing could be driving the allelic dropout. A study on sika deer using ddRAD 
obtained 7,576,300 candidates of the ddRAD loci from all individuals (Ba et al., 2017). After data filtering, $4 \%$ of those loci were shared by more than $75 \%$ of the individuals. Our BOV2A_CE dataset yielded similar results of shared loci with a 90\% cut-off. In terms of raw reads and SNP numbers, Ba et al. (2017) sequenced ca. 34.5 million PE reads per sample, which resulted in 96,000 SNPs in the loci that were shared by more than $75 \%$ of the individuals. In comparison, we sequenced an average of 850,000 PE reads per deer sample, which yielded approximately 115,000 SNPs in the loci that were shared by at least $90 \%$ of the individuals. In this way, despite the need for adjustment in the sequencing effort, our results are comparable to similar studies.

\section{Length of flanking region}

Available RRL methods produce loci with variable length, depending on the cutting enzyme and the chosen sequencing technology (Andrews et al., 2016). Currently, only the latest RADseq version can sequence up to 700 bp fragments (Nelson \& Cresko, 2017). Our method has allowed us to sequence an average of up to $650 \mathrm{bp}$ in wolf LINE, $600 \mathrm{bp}$ in wolf SINE, 500 bp in deer BOV2A, and 650 bp in rat L1, with variable depth of coverage (Figure 3). Length variation is correlated with the fragment length used for library build. In this way, if the aim of the study requires a great extension into the flanking region, it would be crucial to take into consideration the fragmentation level of the starting material.

\section{Ease of adjustment to other taxa}

In this study, we have applied MobiSeq to three different mammal groups, focusing on SINE and LINE retrotransposons. These elements propagate through a copy-and-paste process and, hence, persist in genomic locations after transposition (Kriegs et al., 2006). However, other genomic TEs could be targeted as well. Endogenous retroviruses and LTR 
transposons are flanked by terminal repeats and, in theory, only half of the primers designed for the distal parts of the TEs should extend into the flanking genomic region (the other half would extend into the elements themselves). Yet, a substantial fraction of these elements exist as solo-LTRs (Izsvák, Wang, Singh, Mager, \& Hurst, 2016; Kim, Vanguri, Boeke, Gabriel, \& Voytas, 1998), which will enhance the primer efficiency. Nevertheless, certain TEs have properties that may deem them unsuited for MobiSeq targeting. DNA transposons move through a cut-and-paste mechanism, which will increase allele dropout events. Further, DNA transposons generally display low levels of cis-preference (Arkhipova, 2005), so that older, degenerated elements may continue to be excised and transposed. This means that DNA transposons should provide less well-suited TE targets for MobiSeq. Finding suitable TEs for MobiSeq is a trade-off between elements being young enough for individual loci to possess a conserved site for primer design, and old enough to be near fixation in the population. Elements should further be abundant and dispersed in a highly unbiased fashion. In compact genomes, TEs often display a high degree of insertion preferences, as exemplified by the yeast Schizosaccharomyces pombe, in which the Tf retrotransposons predominantly insert within a few hundred basepairs upstream of open reading frames (Bowen, Jordan, Epstein, Wood, \& Levin, 2003). In larger vertebrate genomes, much less insertion bias is observed, although elements do not insert in an entirely random fashion (International Human Genome Sequencing Consortium, 2001; Mourier, Nielsen, Hansen, \& Willerslev, 2014). Despite these theoretical requirements, our experience with primer design for MobiSeq has been remarkably successful. The fact that the deer primer was designed entirely from published literature without an annotated reference genome available suggests to us that MobiSeq holds great promise for a large range of organisms.

This article is protected by copyright. All rights reserved. 
Despite the benefits of MobiSeq, we caution there are some aspects that need further exploration:

1. One of the limitations of traditional RRL methods is its applicability to noninvasive samples, such as fecal samples. The high proportion of non-host DNA would affect the sequencing efficiency. Chiou and Bergey (2018) suggested a methylation-based enrichment of fecal DNA extracts prior to RRL (RADseq, in their case), as a way to reduce the non-host DNA. As MobiSeq relies on TE-target species specific primers, the presence of non-host DNA should not reduce the efficiency of the method. If MobiSeq can be implemented in non-invasive samples to generate genomic information, it will have a broad applicability in conservation genomics and wildlife forensics at a reduced cost.

2. Our BOV2A_all dataset included specimens from Cervus sp., as well as two fallow deer, which belong to Dama, a different Cervidae genus (Gilbert et al., 2006). Despite their phylogenetic distance, the same TE-target primer allowed us to sequence shared loci and enabled variant calling. Our results indicate that MobiSeq could also be applied to investigate relationships between phylogenetically distant species.

Our study presented MobiSeq, a RRL method that uses TEs as anchor for sequencing extension into the flanking region of these mobile elements. MobiSeq generates high numbers of comparable loci and SNPs across samples at a similar cost compared to other RRL methods ( 4.5 dollars per library build reaction). We also demonstrate the ability of the method to sequence up $650 \mathrm{bp}$ of the flanking region, and its adaptability to target different genome mobile elements. This makes MobiSeq a good alternative to other RRL protocols to perform evolutionary inferences.

This article is protected by copyright. All rights reserved. 


\section{Acknowledgements}

We are grateful to all the people and institutions that have provided samples for this study, specifically: Department of Environment Nunavut, Environment and Natural Resources Northwest Territories, and Lindsey Carmichael and David Coltman at University of Alberta (wolf samples); Frank Zachos (Natural History Museum in Vienna), Meirav Meiri (Tel-Aviv University), Adrian Lister, Ian Barnes, and Richard Sabin (Natural History Museum of London), Kristian Murphy Gregersen (Natural History Museum of Denmark), Rolf Langvatn (University Centre in Svalbard), and Gennady Baryshnikov (Russian Academy of Sciences, Moscow) (deer material). We also thank Lasse Vinner for experimental methodology discussions. Maria Asplund for discussion on data analysis in the early stages of the project. The Danish Advance Technology Foundation. S.G. was funded by EU Marie Słodowska-Curie grant 655732 (Wherewolf).

\section{References}

1. Akagi, K., Li, J., Stephens, R. M., Volfovsky, N., \& Symer, D. E. (2008). Extensive variation between inbred mouse strains due to endogenous L1 retrotransposition. Genome research, 18(6), 869-880. doi: 10.1101/gr.075770.107

2. Andrews, K. R., Good, J. M., Miller, M. R., Luikart, G., \& Hohenlohe, P. A. (2016). Harnessing the power of RADseq for ecological and evolutionary genomics. Nature Reviews Genetics, 17(2), 81. doi: 10.1038/nrg.2015.28

3. Andrews, K. R., \& Luikart, G. (2014). Recent novel approaches for population genomics data analysis. Molecular ecology, 23(7), 1661-1667. doi: 10.1111/mec.12686

4. Arkhipova, I. R. (2005). Mobile genetic elements and sexual reproduction. Cytogenetic and genome research, 110(1-4), 372-382. doi: 10.1159/000084969

5. Ba, H., Jia, B., Wang, G., Yang, Y., Kedem, G., \& Li, C. (2017). Genome-Wide SNP Discovery and Analysis of Genetic Diversity in Farmed Sika Deer (Cervus nippon) in Northeast China Using Double-Digest Restriction Site-Associated DNA Sequencing. G3: Genes, Genomes, Genetics, 7(9), 3169-3176. doi: 10.1534/g3.117.300082

6. Baird, N. A., Etter, P. D., Atwood, T. S., Currey, M. C., Shiver, A. L., Lewis, Z. A., ... \& Johnson, E. A. (2008). Rapid SNP discovery and genetic mapping using sequenced RAD markers. PloS one, 3(10), e3376. doi: 10.1371/journal.pone.0003376

7. Bowen, N. J., Jordan, I. K., Epstein, J. A., Wood, V., \& Levin, H. L. (2003). Retrotransposons and their recognition of pol II promoters: a comprehensive survey of the transposable elements from the complete genome sequence of Schizosaccharomyces pombe. Genome research, 13(9), 1984-1997. doi: 10.1101/gr.1191603

8. Carøe, C., Gopalakrishnan, S., Vinner, L., Mak, S. S., Sinding, M. H. S., Samaniego, J. A., ... \& Gilbert, M. T. P. (2017). Single-tube library preparation for degraded DNA. Methods in Ecology and Evolution, 9(2), 410-419. doi: 10.1111/2041210x. 12871

9. Chénais, B., Caruso, A., Hiard, S., \& Casse, N. (2012). The impact of transposable elements on eukaryotic genomes: from genome size increase to genetic adaptation to stressful environments. Gene, 509(1), 7-15. doi: 10.1016/j.gene.2012.07.042

This article is protected by copyright. All rights reserved. 
10. Chiou, K. L., \& Bergey, C. M. (2018). Methylation-based enrichment facilitates lowcost, noninvasive genomic scale sequencing of populations from feces. Scientific reports, 8(1), 1975. doi: 10.1038/s41598-018-20427-9

11. Cordaux, R., Srikanta, D., Lee, J., Stoneking, M., \& Batzer, M. A. (2007). In search of polymorphic Alu insertions with restricted geographic distributions. Genomics, 90(1), 154-158. doi: 10.1016/j.ygeno.2007.03.010

12. Daley, T., \& Smith, A. D. (2013). Predicting the molecular complexity of sequencing libraries. Nature methods, 10(4), 325. doi: 10.1038/nmeth.2375

13. Davey, J. W., Cezard, T., Fuentes-Utrilla, P., Eland, C., Gharbi, K., \& Blaxter, M. L. (2013). Special features of RAD Sequencing data: implications for genotyping. Molecular ecology, 22(11), 3151-3164. doi: 10.1111/mec.12084

14. Davey, J. W., Hohenlohe, P. A., Etter, P. D., Boone, J. Q., Catchen, J. M., \& Blaxter, M. L. (2011). Genome-wide genetic marker discovery and genotyping using nextgeneration sequencing. Nature Reviews Genetics, 12(7), 499. doi: 10.1038/nrg3012

15. Davey, J. W., \& Blaxter, M. L. (2010). RADSeq: next-generation population genetics. Briefings in functional genomics, 9(5-6), 416-423. doi: 10.1093/bfgp/elr007

16. DePristo, M. A., Banks, E., Poplin, R., Garimella, K. V., Maguire, J. R., Hartl, C., ... \& McKenna, A. (2011). A framework for variation discovery and genotyping using next-generation DNA sequencing data. Nature genetics, 43(5), 491. doi: 10.1038/ng.806

17. Dewannieux, M., Esnault, C., \& Heidmann, T. (2003). LINE-mediated retrotransposition of marked Alu sequences. Nature genetics, 35(1), 41. doi: $10.1038 / n g 1223$

18. Edgar, R. C. (2004). MUSCLE: multiple sequence alignment with high accuracy and high throughput. Nucleic acids research, 32(5), 1792-1797. doi: 10.1093/nar/gkh340

19. Ellegren, H. (2014). Genome sequencing and population genomics in non-model organisms. Trends in ecology \& evolution, 29(1), 51-63. doi: 10.1016/j.tree.2013.09.008

20. Elshire, R. J., Glaubitz, J. C., Sun, Q., Poland, J. A., Kawamoto, K., Buckler, E. S., \& Mitchell, S. E. (2011). A robust, simple genotyping-by-sequencing (GBS) approach for high diversity species. PloS one, 6(5), e19379. doi: 10.1371/journal.pone.0019379

21. Emerson, K. J., Merz, C. R., Catchen, J. M., Hohenlohe, P. A., Cresko, W. A., Bradshaw, W. E., \& Holzapfel, C. M. (2010). Resolving postglacial phylogeography using high-throughput sequencing. Proceedings of the national academy of sciences, 107(37), 16196-16200. doi: 10.1073/pnas.1006538107

22. Ewing, A. D., \& Kazazian, H. H. (2010). High-throughput sequencing reveals extensive variation in human-specific L1 content in individual human genomes. Genome research, 20(9), 1262-1270. doi: 10.1101/gr.106419.110

23. Ewing, A. D., \& Kazazian, H. H. (2011). Whole-genome resequencing allows detection of many rare LINE-1 insertion alleles in humans. Genome research, 21(6), 985-990. doi: 10.1101/gr.114777.110

24. Faircloth, B. C., McCormack, J. E., Crawford, N. G., Harvey, M. G., Brumfield, R. T., \& Glenn, T. C. (2012). Ultraconserved elements anchor thousands of genetic markers spanning multiple evolutionary timescales. Systematic biology, 61(5), 717726. doi: 10.1093/sysbio/sys004

25. Fuentes-Pardo, A. P., \& Ruzzante, D. E. (2017). Whole-genome sequencing approaches for conservation biology: advantages, limitations, and practical recommendations. Molecular ecology. doi: 10.1111/mec.14264

26. Gaither, M. R., Bernal, M. A., Coleman, R. R., Bowen, B. W., Jones, S. A., Simison, W. B., \& Rocha, L. A. (2015). Genomic signatures of geographic isolation and

This article is protected by copyright. All rights reserved. 
natural selection in coral reef fishes. Molecular Ecology, 24(7), 1543-1557. doi: $10.1111 / \mathrm{mec} .13129$

27. Gautier, M., Gharbi, K., Cezard, T., Foucaud, J., Kerdelhué, C., Pudlo, P., ... \& Estoup, A. (2013). The effect of RAD allele dropout on the estimation of genetic variation within and between populations. Molecular Ecology, 22(11), 3165-3178. doi: $10.1111 / \mathrm{mec} .12089$

28. Gilbert, C., Ropiquet, A., \& Hassanin, A. (2006). Mitochondrial and nuclear phylogenies of Cervidae (Mammalia, Ruminantia): systematics, morphology, and biogeography. Molecular phylogenetics and evolution, 40(1), 101-117. doi: 10.1016/j.ympev.2006.02.017

29. Goodwin, S., McPherson, J. D., \& McCombie, W. R. (2016). Coming of age: ten years of next-generation sequencing technologies. Nature Reviews Genetics, 17(6), 333. doi: 10.1038/nrg.2016.49

30. Gopalakrishnan, S., Castruita, J. A. S., Sinding, M. H. S., Kuderna, L. F., Räikkönen, J., Petersen, B., ... \& Hansen, A. J. (2017). The wolf reference genome sequence (Canis lupus lupus) and its implications for Canis spp. population genomics. BMC genomics, 18(1), 495. doi: 10.1186/s12864-017-3883-3

31. Hohenlohe, P. A., Bassham, S., Etter, P. D., Stiffler, N., Johnson, E. A., \& Cresko, W. A. (2010). Population genomics of parallel adaptation in threespine stickleback using sequenced RAD tags. PLoS genetics, 6(2), e1000862. doi: 10.1371/journal.pgen. 1000862

32. International Human Genome Sequencing Consortium. (2001). International human genome sequencing consortium. Nature, 409, 860-921. doi: 10.1037/e634052007-001

33. Izsvák, Z., Wang, J., Singh, M., Mager, D. L., \& Hurst, L. D. (2016). Pluripotency and the endogenous retrovirus HERVH: Conflict or serendipity?. Bioessays, 38(1), 109-117. doi: 10.1002/bies.201500096

34. Kardos, M., Åkesson, M., Fountain, T., Flagstad, Ø., Liberg, O., Olason, P., ... \& Ellegren, H. (2018). Genomic consequences of intensive inbreeding in an isolated wolf population. Nature ecology \& evolution, 2(1), 124. doi: 10.1038/s41559-0170375-4

35. Kazazian, H. H. (2004). Mobile elements: drivers of genome evolution. science, 303(5664), 1626-1632. doi: 10.1126/science.1089670

36. Kim, J. M., Vanguri, S., Boeke, J. D., Gabriel, A., \& Voytas, D. F. (1998). Transposable elements and genome organization: a comprehensive survey of retrotransposons revealed by the complete Saccharomyces cerevisiae genome sequence. Genome research, 8(5), 464-478. doi: 10.1101/gr.8.5.464

37. Konkel, M. K., Wang, J., Liang, P., \& Batzer, M. A. (2007). Identification and characterization of novel polymorphic LINE-1 insertions through comparison of two human genome sequence assemblies. Gene, 390(1), 28-38. doi: 10.1016/j.gene.2006.07.040

38. Korneliussen, T. S., Albrechtsen, A., \& Nielsen, R. (2014). ANGSD: analysis of next generation sequencing data. BMC bioinformatics, 15(1), 356. doi: 10.1186/s12859014-0356-4

39. Kriegs, J. O., Churakov, G., Kiefmann, M., Jordan, U., Brosius, J., \& Schmitz, J. (2006). Retroposed elements as archives for the evolutionary history of placental mammals. PLoS biology, 4(4), e91. doi: 10.1371/journal.pbio.0040091

40. Lah, L., Trense, D., Benke, H., Berggren, P., Gunnlaugsson, P., Lockyer, C., ... \& Siebert, U. (2016). Spatially explicit analysis of genome-wide SNPs detects subtle population structure in a mobile marine mammal, the harbor porpoise. PloS one, 11(10), e0162792. doi: 10.1371/journal.pone.0162792

This article is protected by copyright. All rights reserved. 
41. Lefort, V., Desper, R., \& Gascuel, O. (2015). FastME 2.0: a comprehensive, accurate, and fast distance-based phylogeny inference program. Molecular biology and evolution, 32(10), 2798-2800. doi: 10.1093/molbev/msv150

42. Levy, S., Sutton, G., Ng, P. C., Feuk, L., Halpern, A. L., Walenz, B. P., ... \& Lin, Y. (2007). The diploid genome sequence of an individual human. PLoS biology, 5(10), e254. doi: 10.1371/journal.pbio.0050254

43. Li, H., Handsaker, B., Wysoker, A., Fennell, T., Ruan, J., Homer, N., ... \& Durbin, R. (2009). The sequence alignment/map format and SAMtools. Bioinformatics, 25(16), 2078-2079. doi: 10.1093/bioinformatics/btp352

44. Lindblad-Toh, K., Wade, C. M., Mikkelsen, T. S., Karlsson, E. K., Jaffe, D. B., Kamal, M., ... \& Mauceli, E. (2005). Genome sequence, comparative analysis and haplotype structure of the domestic dog. Nature, 438(7069), 803. doi:10.1038/nature04338

45. Lorenzini, R., \& Garofalo, L. (2015). Insights into the evolutionary history of Cervus (Cervidae, tribe Cervini) based on Bayesian analysis of mitochondrial marker sequences, with first indications for a new species. Journal of Zoological Systematics and Evolutionary Research, 53(4), 340-349. doi:10.1111/jzs.12104

46. Luan, D. D., Korman, M. H., Jakubczak, J. L., \& Eickbush, T. H. (1993). Reverse transcription of R2Bm RNA is primed by a nick at the chromosomal target site: a mechanism for non-LTR retrotransposition. Cell, 72(4), 595-605. doi: 10.1016/00928674(93)90078-5

47. Maehara, K., \& Ohkawa, Y. (2015). agplus: a rapid and flexible tool for aggregation plots. Bioinformatics, 31(18), 3046-3047. doi: 10.1093/bioinformatics/btv322

48. Mak, S. S. T., Gopalakrishnan, S., Carøe, C., Geng, C., Liu, S., Sinding, M. H. S., ... \& Germonpré, M. (2017). Comparative performance of the BGISEQ-500 vs Illumina HiSeq2500 sequencing platforms for palaeogenomic sequencing. GigaScience, 6(8), 1-13. doi: 10.1093/gigascience/gix049

49. Meyer, M., \& Kircher, M. (2010). Illumina sequencing library preparation for highly multiplexed target capture and sequencing. Cold Spring Harbor Protocols, 2010(6), pdb-prot5448. doi: 10.1101/pdb.prot5448

50. Mills, R. E., Bennett, E. A., Iskow, R. C., Luttig, C. T., Tsui, C., Pittard, W. S., \& Devine, S. E. (2006). Recently mobilized transposons in the human and chimpanzee genomes. The American Journal of human genetics, 78(4), 671-679. doi: $10.1086 / 501028$

51. Mourier, T., Nielsen, L. P., Hansen, A. J., \& Willerslev, E. (2014). Transposable elements in cancer as a by-product of stress-induced evolvability. Frontiers in genetics, 5, 156. doi: 10.3389/fgene.2014.00156

52. Nelson, T. C., \& Cresko, W. A. (2018). Ancient genomic variation underlies repeated ecological adaptation in young stickleback populations. Evolution Letters, 2(1), 9-21. doi: 10.1101/167981

53. Nilsson, M. A., Klassert, D., Bertelsen, M. F., Hallström, B. M., \& Janke, A. (2012). Activity of ancient RTE retroposons during the evolution of cows, spiral-horned antelopes, and Nilgais (Bovinae). Molecular biology and evolution, 29(10), 28852888. doi: $10.1093 / \mathrm{molbev} / \mathrm{mss} 158$

54. Onami, J. I., Nikaido, M., Mannen, H., \& Okada, N. (2007). Genomic expansion of the Bov-A2 retroposon relating to phylogeny and breed management. Mammalian Genome, 18(3), 187-196. doi: 10.1007/s00335-007-9000-1

55. Peterson, B. K., Weber, J. N., Kay, E. H., Fisher, H. S., \& Hoekstra, H. E. (2012). Double digest RADseq: an inexpensive method for de novo SNP discovery and

This article is protected by copyright. All rights reserved. 
genotyping in model and non-model species. PloS one, 7(5), e37135. doi: 10.1371/journal.pone.0037135

56. Pilot, M., Jedrzejewski, W., Branicki, W., Sidorovich, V. E., Jedrzejewska, B., Stachura, K., \& Funk, S. M. (2006). Ecological factors influence population genetic structure of European grey wolves. Molecular ecology, 15(14), 4533-4553. doi: 10.1111/j.1365-294x.2006.03110.x

57. Quinlan, A. R., \& Hall, I. M. (2010). BEDTools: a flexible suite of utilities for comparing genomic features. Bioinformatics, 26(6), 841-842. doi: 10.1093/bioinformatics/btq033

58. Rat Genome Sequencing Project Consortium. (2004). Genome sequence of the Brown Norway rat yields insights into mammalian evolution. Nature, 428(6982), 493. doi: 10.1038/nature02426

59. Ray, D. A., Feschotte, C., Pagan, H. J., Smith, J. D., Pritham, E. J., Arensburger, P., ... \& Craig, N. L. (2008). Multiple waves of recent DNA transposon activity in the bat, Myotis lucifugus. Genome Research. doi: 10.1101/gr.071886.107

60. Rutledge, L. Y., Devillard, S., Boone, J. Q., Hohenlohe, P. A., \& White, B. N. (2015). RAD sequencing and genomic simulations resolve hybrid origins within North American Canis. Biology letters, 11(7), 20150303. doi: 10.1098/rsbl.2015.0303

61. Sánchez Barreiro, F., Vieira, F. G., Martin, M. D., Haile, J., Gilbert, M. T. P., \& Wales, N. (2017). Characterizing restriction enzyme-associated loci in historic ragweed (Ambrosia artemisiifolia) voucher specimens using custom-designed RNA probes. Molecular ecology resources, 17(2), 209-220. doi: 10.1111/1755-0998.12610

62. Schmid, S., Genevest, R., Gobet, E., Suchan, T., Sperisen, C., Tinner, W., \& Alvarez, N. (2017). HyRAD-X, a versatile method combining exome capture and RAD sequencing to extract genomic information from ancient DNA. Methods in Ecology and Evolution, 8(10), 1374-1388. doi: 10.1111/2041-210x.12785

63. Schubert, M., Ermini, L., Der Sarkissian, C., Jónsson, H., Ginolhac, A., Schaefer, R., ... \& Willerslev, E. (2014). Characterization of ancient and modern genomes by SNP detection and phylogenomic and metagenomic analysis using PALEOMIX. Nature protocols, 9(5), 1056. doi: 10.1038/nprot.2014.063

64. Schweyen, H., Rozenberg, A., \& Leese, F. (2014). Detection and removal of PCR duplicates in population genomic ddRAD studies by addition of a degenerate base region (DBR) in sequencing adapters. The Biological Bulletin, 227(2), 146-160. doi: 10.1086/bblv227n2p146

65. Sela, N., Mersch, B., Hotz-Wagenblatt, A., \& Ast, G. (2010). Characteristics of transposable element exonization within human and mouse. PLoS One, 5(6), e10907. doi: 10.1371/journal.pone.0010907

66. Skovrind, M., Olsen, M. T., Vieira, F. G., Pacheco, G., Carl, H., Gilbert, M. T. P., \& Møller, P. R. (2016). Genomic population structure of freshwater-resident and anadromous ide (Leuciscus idus) in north-western Europe. Ecology and evolution, 6(4), 1064-1074. doi: 10.1002/ece3.1909

67. Smit, A. F. A., Hubley, R., \& Green, P. (2013). 2013-2015. RepeatMasker Open-4.0. doi: 10.1002/0471650129.dob0616

68. Sotero-Caio, C. G., Platt, R. N., Suh, A., \& Ray, D. A. (2017). Evolution and diversity of transposable elements in vertebrate genomes. Genome biology and evolution, 9(1), 161-177. doi: 10.1093/gbe/evw264

69. Speir, M. L., Zweig, A. S., Rosenbloom, K. R., Raney, B. J., Paten, B., Nejad, P., ... \& Heitner, S. (2015). The UCSC genome browser database: 2016 update. Nucleic acids research, 44(D1), D717-D725. doi:10.1093/nar/gkg129

This article is protected by copyright. All rights reserved. 
70. Stamatakis, A. (2014). RAxML version 8: a tool for phylogenetic analysis and postanalysis of large phylogenies. Bioinformatics, 30(9), 1312-1313. doi: 10.1093/bioinformatics/btu033

71. Tang, Z., Steranka, J. P., Ma, S., Grivainis, M., Rodić, N., Huang, C. R. L., ... \& Burns, K. H. (2017). Human transposon insertion profiling: Analysis, visualization and identification of somatic LINE-1 insertions in ovarian cancer. Proceedings of the National Academy of Sciences, 114(5), E733-E740. doi: 10.1073/pnas.1619797114

72. Tubio, J. M., Li, Y., Ju, Y. S., Martincorena, I., Cooke, S. L., Tojo, M., ... \& Menzies, A. (2014). Extensive transduction of nonrepetitive DNA mediated by L1 retrotransposition in cancer genomes. Science, 345(6196), 1251343. doi: 10.1126/science. 1251343

73. Vieira, F. G., Lassalle, F., Korneliussen, T. S., \& Fumagalli, M. (2015). Improving the estimation of genetic distances from Next-Generation Sequencing data. Biological journal of the Linnean Society, 117(1), 139-149. doi: 10.1111/bij.12511

74. Wagner, C. E., Keller, I., Wittwer, S., Selz, O. M., Mwaiko, S., Greuter, L., ... \& Seehausen, O. (2013). Genome-wide RAD sequence data provide unprecedented resolution of species boundaries and relationships in the Lake Victoria cichlid adaptive radiation. Molecular ecology, 22(3), 787-798. doi: 10.1111/mec.12023

75. Yohn, C. T., Jiang, Z., McGrath, S. D., Hayden, K. E., Khaitovich, P., Johnson, M. E., ... \& Eichler, E. E. (2005). Lineage-specific expansions of retroviral insertions within the genomes of African great apes but not humans and orangutans. PLoS biology, 3(4), e110. doi: 10.1371/journal.pbio.0030110

\section{Author contributions}

A.J.H., T.M, M.T.P.G. and M.B conceived the ideas and designed methodology. D.A.P. and A.A.N. conducted the laboratory work in the initial phases of method development. C.C. and A.R.I. optimized, implemented the laboratory method, and conducted laboratory work in the rat and deer datasets. M.H.S.S. performed the sampling and curation of the wolf material. L.B.P. and C.N.N. conducted laboratory work in the wolf dataset. M. F. R. performed rat dissection and tissue sampling. S.G., T.R., T.M., and A.R.I. conducted data analyses. Z.L. and B.P. generated and assembled the red deer genome. A.R.I. wrote the manuscript with input from all the authors. All authors read the final draft and approved it for publication.

\section{Data Accessibility}

The data is accessible at the Electronic Research Data Archive at the University of Copenhagen (UCPH ERDA): https://sid.erda.dk/wsgi-bin/ls.py?share_id=bTgYwewBR9. All the scripts used for processing the data and generating the plots included in the main and supplementary material are available on GitHub at https://github.com/shyamsg/MobiSeq.

This article is protected by copyright. All rights reserved. 


\section{Figures and tables:}

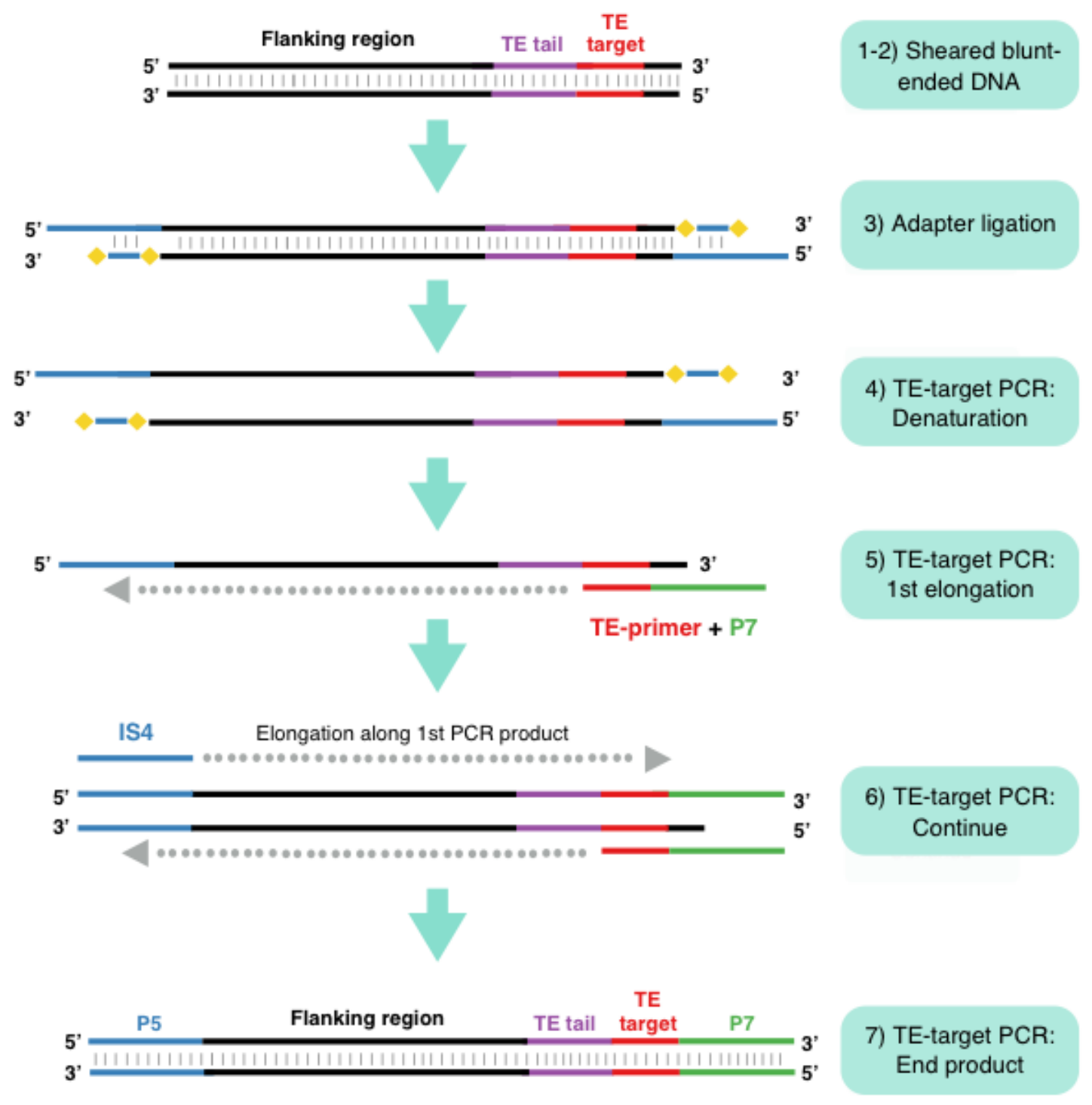

Figure 1. Schematic overview of the protocol. 1) Sample DNA is sheared (if required) into 600 - 800 bp average size fragments. 2) During blunt-end repair, overhanging 5' and 3' ends are filled in or removed by T4 DNA polymerase. 5' phosphates are attached using T4 polynucleotide kinase. 3) Double-stranded MP5 adapters are ligated to the fragment by T4 DNA Ligase. Adapters do not carry 5' phosphates and therefore only one strand is ligated to the fragments. 4) TE-target PCR is performed using forward IS4 primer and the TE-fusion primer. TE-target PCR will also result in the incorporation of P7 sequencing adapter. 5) PCR elongation will only occur upstream of the 3' end of the TE-target primer. 6) The end product of the TE-target PCR will be the TE-target sequence, a TE tail and the flanking genomic region. It will also contain Illumina sequencing adapters (P5 and P7). This PCR product can be indexed using single or dual indexing primers, then pooled for sequencing with other samples. Yellow diamonds represent mP5 adapter modifications.

This article is protected by copyright. All rights reserved. 


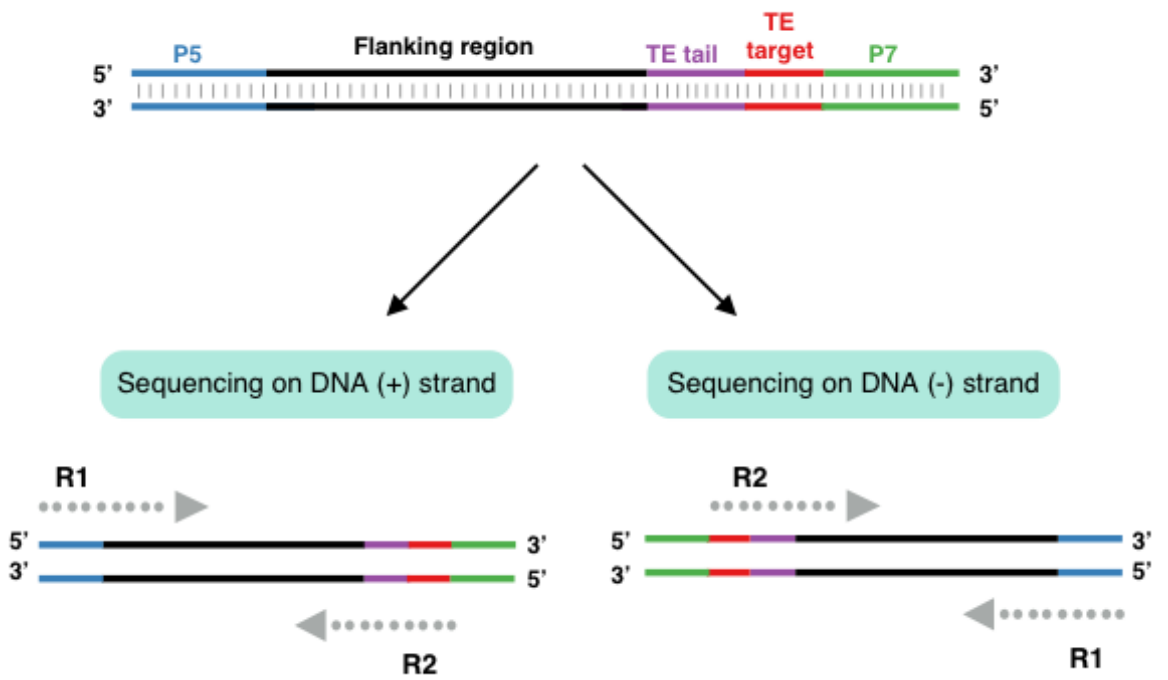

Figure 2. Sequencing architecture of Transposable Element (TE) flanking region in the (+) and (-) strand. The TE-target primer will always be at the 5'end of Read 2 (R2). Sequencing into the flanking will only happen from the 3' end into the flanking region. The start of Read 1 (R1) will be variable, as DNA was random sheared, which allows the identification of PCR duplicates.

This article is protected by copyright. All rights reserved. 

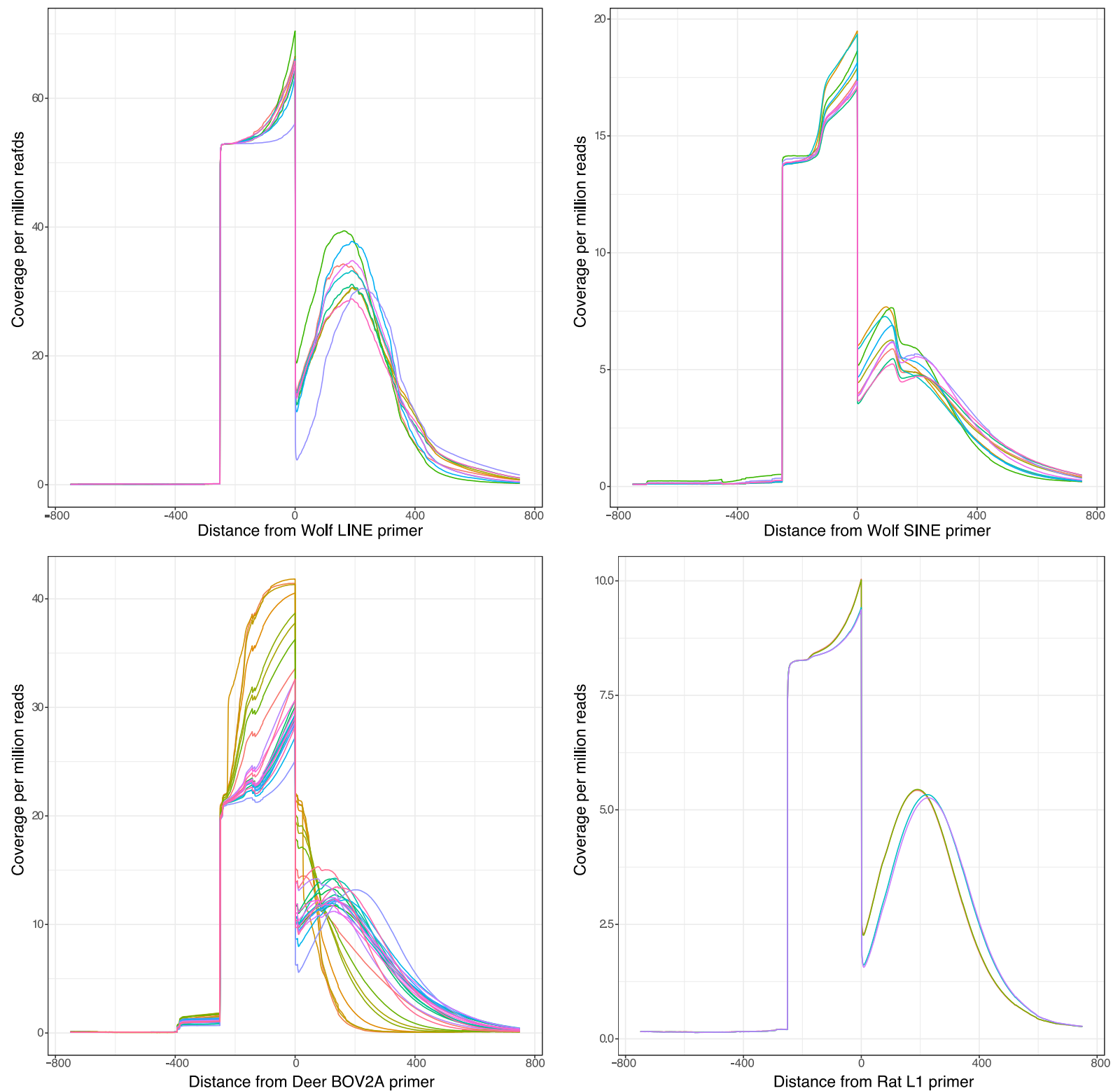

Figure 3. Aggregate coverage plots generated using agplus. Y-axis represents coverage per million reads; coverage was normalized to mitigate read number differences between samples. X-axis represents distance from TE-target primer. Zero represents the start of the TE-target primer. Each line represents a single individual.

This article is protected by copyright. All rights reserved. 

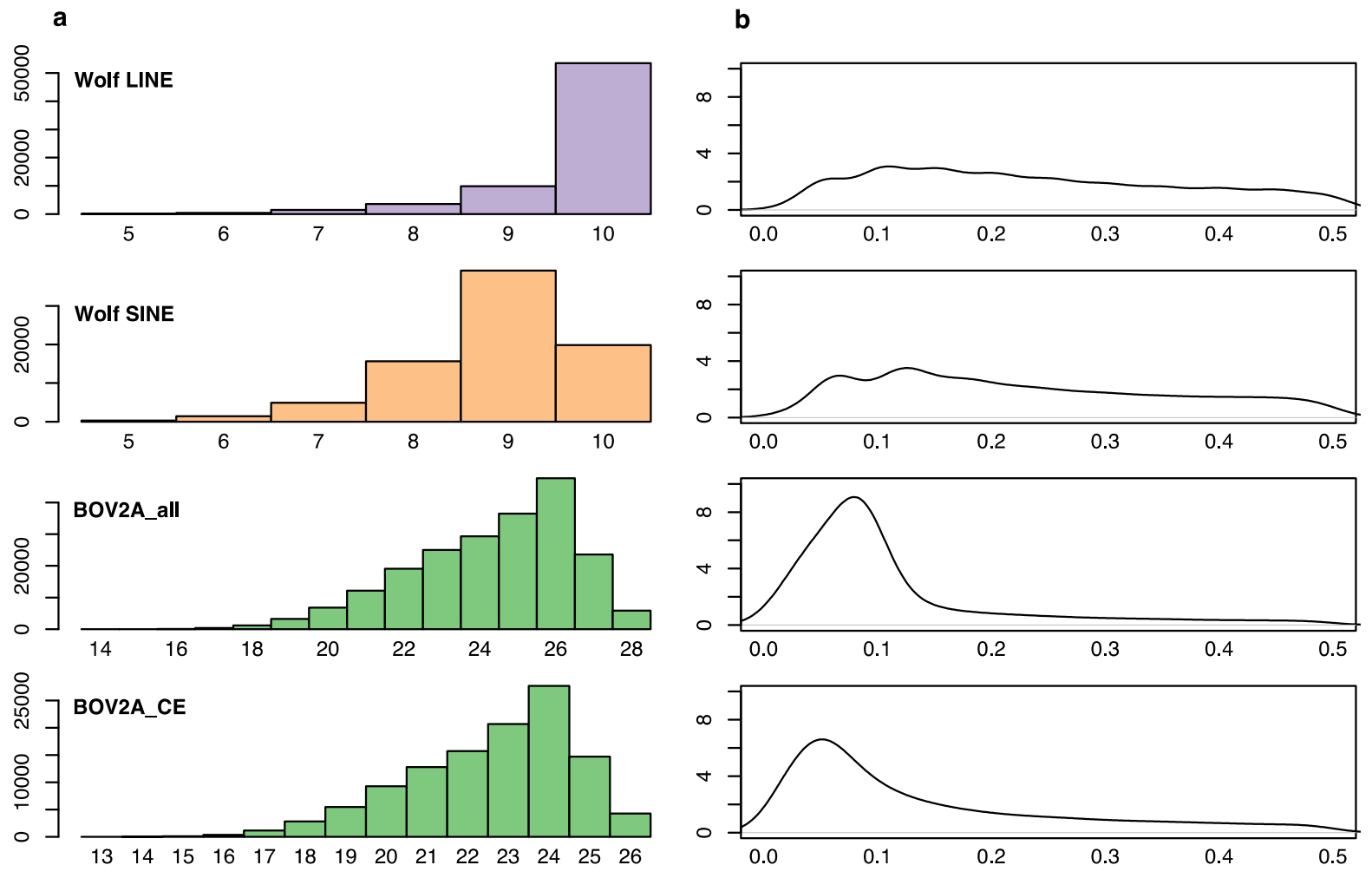

Number of individuals

Density of minor allele frequencies

(Bandwidth $=0.02$ )

Figure 4. (a) Histogram of the number of SNPs (with coverage > 3) vs. the number of individuals with data for a given position. (b) Distribution of minor allele frequencies. The yaxis represents the density of minor allele frequencies.

This article is protected by copyright. All rights reserved. 

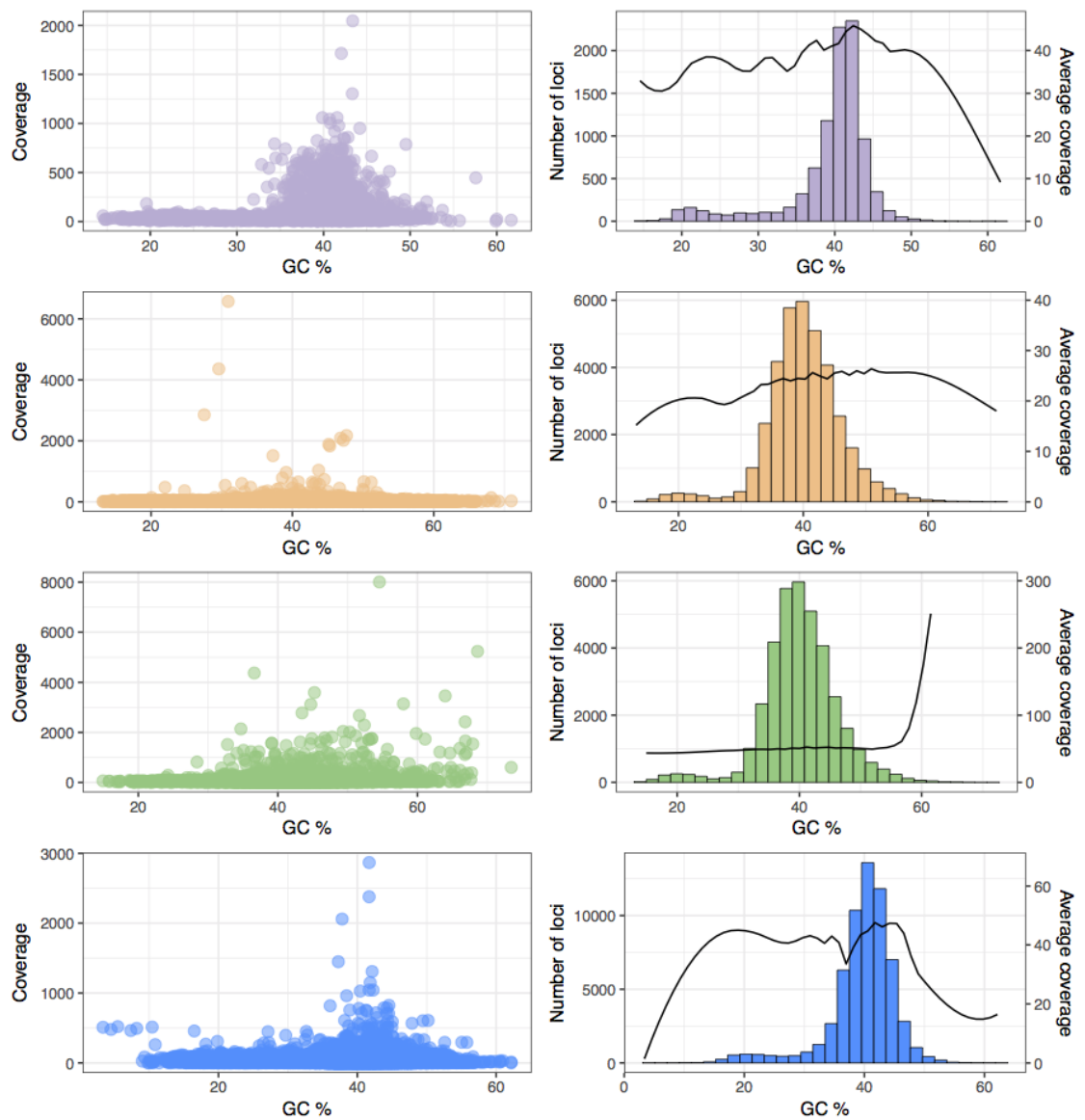

Figure 5. GC content (\%) variation and coverage. Left panel represents average coverage expressed as average number of reads across loci versus GC (\%). Right panel represents average $\mathrm{GC}$ content $(\%)$ in the different loci as a histogram, as well as the average coverage variation at those loci. Colours indicate the different datasets: lilac $=$ Wolf_LINE; orange $=$ Wolf_SINE; green = BOV2A_all; blue = Rat_L1.

This article is protected by copyright. All rights reserved. 

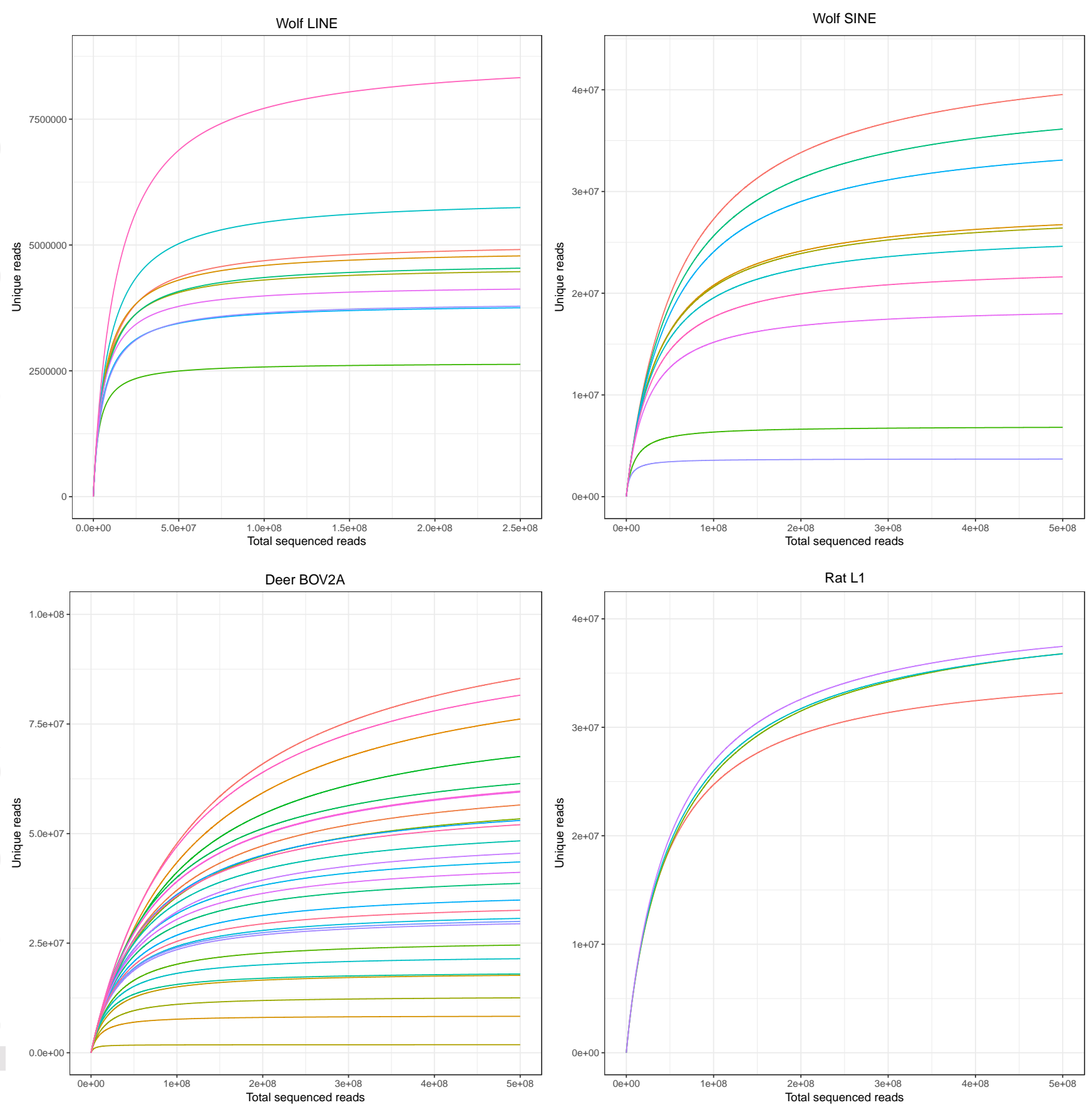

Figure 6. Plotted values generated by PRESEQ showing estimated number of distinct reads for each TE-target primer. Vertical dashed lines represent the average sequencing reads generated for each dataset.

This article is protected by copyright. All rights reserved. 
Table 1. Average sequencing and mapping statistics per TE-target primer pair. More detailed sequencing and mapping statistics per sample can be found in Table S3 (Supporting Information).

\begin{tabular}{|c|c|c|c|c|c|c|c|c|}
\hline Average & $\begin{array}{l}\text { Total } \\
\text { sequenced } \\
\text { reads }\end{array}$ & $\begin{array}{l}\text { Reads with } \\
\text { valid primer }\end{array}$ & $\begin{array}{l}\text { Reads } \\
\text { after adapter } \\
\text { removal }\end{array}$ & $\begin{array}{l}\text { Reads } \\
\text { mapping }\end{array}$ & $\begin{array}{l}\text { Reads } \\
\text { mapping \% }\end{array}$ & $\begin{array}{l}\text { Reads } \\
\text { mapping } \\
\text { after } \\
\text { duplicate } \\
\text { removal }\end{array}$ & $\begin{array}{l}\text { Read } \\
\text { mapping \% } \\
\text { after } \\
\text { duplicate } \\
\text { removal }\end{array}$ & $\begin{array}{l}\text { Clonality } \\
\%\end{array}$ \\
\hline Wolf_LINE & 431,916 & 405,378 & 405,262 & 405,011 & 99.95 & 351,119 & 99.99 & 11.7 \\
\hline Wolf_SINE & 959,580 & 929,932 & 928,504 & 928,540 & 99.90 & 894,928 & 99.99 & 3.3 \\
\hline Deer_BOV2A & $1,695,644$ & $1,593,592$ & $1,590,334$ & $1,589,287$ & 99.96 & $1,507,708$ & 99.98 & 4.5 \\
\hline Rat_L1 & $7,279,504$ & $6,941,756$ & $6,941,686$ & $6,930,739$ & 99.84 & $6,840,096$ & 99.99 & 1.3 \\
\hline
\end{tabular}

Table 2. Total number of TE loci identified in our samples and number of loci identified in $90 \%$ of the samples within each dataset.

TE-target $\quad$ All loci $\quad 90 \%$ cut-off loci

\begin{tabular}{|rcr|}
\hline Wolf_LINE & 33,761 & 9,472 \\
\hline Wolf_SINE & 191,751 & 36,596 \\
\hline BOV2A_all & 749,606 & 23,208 \\
\hline BOV2A_CE & 653,088 & 24,134 \\
\hline Rat_L1 & 270,726 & 61,845 \\
\hline
\end{tabular}

This article is protected by copyright. All rights reserved. 